\title{
Finite time synchronization problems of delayed complex networks with stochastic perturbations
}

\author{
Wenxia Cui ${ }^{1,2^{*}}$, Jian-an Fang ${ }^{2}$ and Shengchao Su ${ }^{2,3}$
}

\section{"Correspondence:} cuiwx423@163.com

'College of Fundamental Studies, Shanghai University of Engineering Science, Shanghai, 201620, China ${ }^{2}$ College of Information Science and Technology, Donghua University, Shanghai, 201620, China

Full list of author information is available at the end of the article

\begin{abstract}
The paper is concerned with the finite-time synchronization problem of delayed complex networks with stochastic perturbations. Based on the finite-time stability theorem, some sufficient conditions are obtained to ensure finite-time synchronization for the Markovian jump complex networks with time delays and partially unknown transition rates. Finally, the effectiveness of the proposed method is demonstrated by illustrative examples.
\end{abstract}

Keywords: finite-time; synchronization; stochastic perturbations; complex networks; time delays

\section{Introduction}

Over the past decades, the dynamics analysis of complex networks has witnessed rapidly growing research interests since the pioneering work of Watts and Strogatz [1]. On the one hand, complex networks exist in our daily life with examples including the Internet, the World Wide Web, food webs, electric power grids, cellular and metabolic networks, etc. [2]. And on the other hand, the dynamical behaviors of complex networks have found numerous applications in various fields such as physics, technology, and the life sciences [3]. In fact, synchronization is a basic motion in nature that has been studied for a long time [4-6]. Recently, synchronization of complex networks has received increasing research attention [7-10].

It is important to note that most of the above research results on network synchronization are based on the asymptotic process of an infinite time. That is, network synchronization only occurs when the time tends to infinity. Thus in theory, it is impossible for a network to achieve synchronization in a limited time. However, in actual physical or engineering systems, complex networks usually achieve synchronization state in a limited time, which is finite-time synchronization. On the one hand, in the existing literature on finite time synchronization is not treated often. And on the other hand, finite-time synchronization is a very important bridge for a complex network to succeed in the actual application. In addition, more and more researchers begin to realize the important role of finite-time synchronization, and there are some related research results [11-14].

Time delays often occur in complex networks because of the limited speed of signals traveling through the links [8] and the frequently delayed couplings in biological neural networks, gene regulatory networks, communication networks, and electrical power grids

O2014 Cui et al:; licensee Springer. This is an Open Access article distributed under the terms of the Creative Commons Attribution License (http://creativecommons.org/licenses/by/2.0), which permits unrestricted use, distribution, and reproduction in any medium, provided the original work is properly cited. 
$[9,10]$. It has been well known that time delays can cause complex dynamics such as periodic or quasi-periodic motions, Hopf bifurcation, and higher-dimensional chaos. It should be noted that [11,12] and [14] did not consider the time-delay problem. Although there are some works that have been reported on the finite synchronization on delayed networks systems, they are mainly concerned with the finite-time boundedness [15]. It is checked that the finite-time boundedness is conservative rather than the finite time convergence. In addition, stochastic perturbation becomes one of the main sources for causing instability and poor performance of networks [9].

In reality, it has been revealed that a neural network sometimes has finite modes, so that switching from one to another at different times may occur $[10,16]$. And such a switching (or jumping) can be governed by a Markovian chain [17, 18]. This is partly because a Markovian jump is a suitable mathematical pattern to represent a class of complex networks subject to random abrupt variations in the structures [18]. Moreover, Markovian jump complex networks can be regarded as a special class of stochastic network systems. So a great number of significant results on synchronization of Markovian switching networked systems have been available in the literature $[8,10,16]$. Unfortunately, almost all of the above mentioned works on the synchronization problem of complex networks are built upon the assumption that switching probabilities are known precisely. However, in most cases the transition probabilities of Markovian jump systems or networks are not exactly known [19-21]. Moreover, the estimated values of the mode transition rates may also lead to instability or at least degraded system performance as the partially unknown mode transition rates in system matrices do [22]. Some extended results concerning the uncertain transition probabilities have been reported in [22, 23]. However, such uncertainties require the knowledge of a bound or structure of uncertainties, which is conservative to some extent.

Although the finite-time stability or stabilization problems of the control systems has received much attention [15, 24, 25], finite-time synchronization of the delayed complex networks has attracted comparatively less attention primarily due to the lack of an appropriate control method, and secondly due to the difficulty residing in the mathematical derivation. Besides, how to tackle the coexistence of finite-time synchronization and the other two typical networked-induced constraints, stochastic disturbance and time delays in Markovian jump complex networks with partially unknown transition rates, still remains open.

In this paper, finite-time synchronization problems are studied for the delayed complex networks with stochastic perturbations and incomplete description of their transition rates. The main features of this paper are twofold: (1) Based on the finite-time stability theorem, some sufficient conditions are obtained to ensure finite-time synchronization for the Markovian jump complex networks with time delays and partially unknown transition rates. (2) For finite-time synchronization research, the model in this paper is more practical, because the network model involves time delays and stochastic perturbations.

Notation Throughout this paper, $R^{n}$ and $R^{n \times m}$ denote, respectively, the $n$-dimensional Euclidean space and the set of all $n \times m$ real matrices. The superscript ' $T$ ' denotes the transpose and the notation $X \geq Y$ (respectively, $X>Y$ ) where $X$ and $Y$ are symmetric matrices, means that $X-Y$ is positive semi-definite (respectively, positive definite); $I$ is the identity matrix with compatible dimension. $\|\cdot\|$ refers to the Euclidean vector norm; the notation $A \otimes B$ stands for the Kronecker product of matrices $A$ and $B$. If $A$ is a matrix, 
$\lambda_{\min }(\cdot)$ denotes the minimum eigenvalue. $\operatorname{diag}\{\cdots\}$ stands for a block-diagonal matrix $\mathbf{E}$. $\mathbf{E}[x]$ means the expectation of the random variable $x$. Matrices, if their dimensions are not explicitly stated, are assumed to be compatible for algebraic operations.

\section{System description and preliminaries}

Consider a Markovian switching time-delay complex network composed of $N$ identical nodes with diffusively couplings, in which each node is an $n$-dimensional delayed dynamical system described by

$$
\begin{aligned}
d x_{i}(t)= & {\left[f\left(x_{i}(t), x_{i}(t-\tau)\right)+c \sum_{j=1}^{N} a_{i j}(r(t)) \Gamma x_{j}(t)+u_{i}(t)\right] d t } \\
& +h_{i}(x(t), x(t-\tau), r(t)) d \omega_{i}(t), \quad i=1,2, \ldots, N
\end{aligned}
$$

where $x_{i}(t)=\left[x_{i 1}(t), x_{i 2}(t), \ldots, x_{i n}(t)\right]^{T} \in R^{n}$ represents the state vector of the $i$ th node, $\tau>0$ is the time delay of node $i, f\left(x_{i}(t), x_{i}(t-\tau)\right)=\left(f_{1}\left(x_{i}(t), x_{i}(t-\tau)\right), f_{2}\left(x_{i}(t), x_{i}(t-\right.\right.$ $\left.\tau)), \ldots, f_{n}\left(x_{i}(t), x_{i}(t-\tau)\right)\right)^{T} \in R^{n}$ is a continuous vector-valued function, we denote $h_{i}(x(t)$, $x(t-\tau), r(t))=h_{i}\left(x_{1}(t), x_{2}(t), \ldots, x_{N}(t), x_{1}(t-\tau), x_{2}(t-\tau), \ldots, x_{N}(t-\tau), r(t)\right) \in R^{n \times n}$ is the unknown diffusive coupling matrix. Here, $A(r(t))=\left(a_{i j}(r(t))\right)_{N \times N}$ describes the linear coupling configuration of the network at time $t$ at mode $r(t)$, and is defined as $a_{i j}(r(t)) \geq 0$, for $i \neq j, a_{i i}(r(t))=-\sum_{j=1, j \neq i}^{N} a_{i j}(r(t)), i=1,2, \ldots, N . \Gamma=\operatorname{diag}\left\{\gamma_{1}, \gamma_{2}, \ldots, \gamma_{n}\right\}$ is the weighted inner-coupling matrix between nodes, which is positive definite. $u_{i}(t)$ denotes the control input on the node $i, \omega_{i}(t)=\left(\omega_{i 1}(t), \omega_{i 2}(t), \ldots, \omega_{i n}(t)\right)^{T}$ is a bounded vector-form Weiner process that is independent of the Markovian chain $r(\cdot)$, satisfying

$$
\mathbf{E}\left[\omega_{i j}(t)\right]=0, \quad \mathbf{E}\left[\omega_{i j}^{2}(t)\right]=1, \quad \mathbf{E}\left[\omega_{i j}(t) \omega_{i j}(s)\right]=0 \quad(s \neq t) .
$$

Let $\{r(t), t \geq 0\}$ be a right-continuous Markovian process that describes the evolution of the modes at time $t$ and takes values in the finite space $S=\{1,2, \ldots, r\}$, with a generator $\Pi=\left\{\pi_{\hat{i}}\right\}, \hat{i}, \hat{j} \in S$ given by

$$
P\{r(t+\Delta t)=\hat{j}: r(t)=\hat{i}\}= \begin{cases}\pi_{\hat{i}} \Delta t+o(\Delta t), & \text { if } \hat{j} \neq \hat{i}, \\ 1+\pi_{\hat{i} j} \Delta t+o(\Delta t), & \text { if } \hat{j}=\hat{i},\end{cases}
$$

with $\Delta t>0$, and $\lim _{\Delta t \rightarrow 0}(o(\Delta t) / \Delta t)=0$. Here, $\pi_{\hat{i}} \geq 0$ is the transition rate from $\hat{i}$ to $\hat{j}$ if $\hat{j} \neq \hat{i}$, while $\pi_{\hat{i} i}=-\sum_{\hat{j}=1, \hat{j} \neq \hat{i}}^{N} \pi_{\hat{i}}$.

In this paper, the transition rates of the jumping process are considered to be partly accessed. For example, the transition rate matrix for network system (1) with $r$ operation modes can be expressed as

$$
\left[\begin{array}{cccc}
\pi_{11} & ? & \ldots & ? \\
\pi_{21} & ? & \ldots & \pi_{2 r} \\
\vdots & \vdots & \ddots & \vdots \\
? & \pi_{r 2} & \ldots & \pi_{r r}
\end{array}\right]
$$

where '?' represents the unknown transition rate. For notational clarity, $\forall \hat{i} \in S$, we denote $S=S_{1}^{\hat{i}} \cup S_{2}^{\hat{i}}$ with $S_{1}^{\hat{i}}=\left\{\hat{j} \mid \pi_{\hat{i}}\right.$ is known $\}, S_{2}^{\hat{i}}=\left\{\hat{j} \mid \pi_{\hat{i} \hat{j}}\right.$ is unknown $\}$. 
The isolated node (or the uncoupled node) of network (1) is given by

$$
\dot{s}(t)=f(s(t), s(t-\tau), t)
$$

where $s(t)=\left(s_{1}(t), s_{2}(t), \ldots, s_{n}(t)\right)^{T} \in R^{n}, s(t-\tau)=\left(s_{1}(t-\tau), s_{2}(t-\tau), \ldots, s_{n}(t-\tau)\right)^{T} \in R^{n}$.

The diffusive couplings mean that the coupled networks (1) are decoupled when the systems are synchronized. So, the coupling terms satisfy $\sum_{j=1}^{N} a_{i j}(r(t)) \Gamma s(t)=0, h_{i}(s(t), s(t-$ $\tau), r(t))=0_{n \times n}$, where $0_{n \times n}$ denotes zero matrix of $n$ dimension.

Our control objective is to finite-timely synchronize complex network (1) to the homogeneous trajectory (3). To reduce the number of controllers, we can adopt the control nodes set $J=\{l, l+1, \ldots, N\}$, where $1<l<N$. That is, by adding the suitable designed feedback controller to complex networks (1), there exists a constant $t^{*}>0\left(t^{*}\right.$ depends on the initial state vector value $\left.x(0)=\left(x_{1}^{T}(0), x_{2}^{T}(0), \ldots, x_{N}^{T}(0)\right)^{T}\right)$, for any $t \geq t^{*}$, such that

$$
x_{1}(t)=x_{2}(t)=\cdots=x_{N}(t)=s(t) .
$$

Definition 1 The Markovian jump complex network (1) is said to be synchronization in finite time, if there exists a constant $t^{*}>0\left(t^{*}\right.$ depends on the initial state vector value $\left.x(0)=\left(x_{1}^{T}(0), x_{2}^{T}(0), \ldots, x_{N}^{T}(0)\right)^{T}\right)$, for any $t \geq t^{*}$, such that

$$
\lim _{t \rightarrow t^{*}} \sum_{i=1}^{N} \mathbf{E}\left\|x_{i}(t)-s(t)\right\|=0,
$$

where $s(t)=\left(s_{1}(t), s_{2}(t), \ldots, s_{n}(t)\right)^{T} \in R^{n}$ is the particular solution of the system (3).

Remark 1 In order to fit the model here, we give the definition of $[13-15,24,25]$ as follows:

The complex network (1) is said to be finite-time synchronization (finite-time boundedness) with respect to $\left(c_{1}, c_{2}, T\right)$ with $c_{1}<c_{2}$, if for

$\sum_{1 \leq i<j \leq M}\left\|x_{i}(0)-x_{j}(0)\right\|^{2} \leq c_{1}$, one has

$$
\sum_{1 \leq i<j \leq M} \mathbf{E}\left\{\left\|x_{i}(t)-x_{j}(t)\right\|^{2}\right\}<c_{2}, \quad \forall t \in[0, T], i, j=1,2, \ldots, M
$$

Obviously, there are differences between the above two definitions, the definition of [13$15,24,25]$ has more conservatism.

We need the following assumption to study the finite-time synchronization of the complex network (1).

Assumption 1 There exist two constant matrices $\Theta=\left(\theta_{i j}\right)_{n \times n}$ and $\Phi=\left(\varphi_{i j}\right)_{n \times n}$, in which $\theta_{i j} \geq 0, \varphi_{i j} \geq 0$, such that

$$
\begin{aligned}
& \left|f_{i}(t, x(t), x(t-\tau))-f_{i}(t, y(t), y(t-\tau))\right| \\
& \quad \leq \sum_{j=1}^{n}\left(\theta_{i j}\left|x_{j}(t)-y_{j}(t)\right|+\varphi_{i j}\left|x_{j}(t-\tau)-y_{j}(t-\tau)\right|\right),
\end{aligned}
$$

$\forall x=\left(x_{1}, x_{2}, \ldots, x_{n}\right)^{T} \in R^{n}, y=\left(y_{1}, y_{2}, \ldots, y_{n}\right)^{T} \in R^{n}, i=1,2, \ldots, n$. 
By Assumption 1, define the following parameters:

$$
\bar{a}=\max _{1 \leq \mu \leq n} \sum_{\nu=1}^{n}\left(\theta_{\mu \nu}^{2 \epsilon}+\varphi_{\mu \nu}^{2 \epsilon}+\theta_{\nu \mu}^{2(1-\epsilon)}\right), \quad \bar{b}=\max _{1 \leq \mu \leq n} \sum_{\nu=1}^{n} \varphi_{\nu \mu}^{2(1-\epsilon)}
$$

where $\epsilon \in[0,1]$.

Assumption 2 There exist nonnegative constants $\xi_{i j}^{\hat{i}}, \eta_{i j}^{\hat{i}}, i, j=1,2, \ldots, N$, such that

$$
\begin{gathered}
\operatorname{trace}\left(\tilde{h}_{i}^{T}(e(t), e(t-\tau), r(t)) \tilde{h}_{i}(e(t), e(t-\tau), r(t))\right) \\
\quad \leq \sum_{j=1}^{N}\left(\xi_{i j}^{\hat{i}} e_{i}^{T}(t) e_{i}(t)+\eta_{i j}^{\hat{i}} e_{i}^{T}(t-\tau) e_{i}(t-\tau)\right)
\end{gathered}
$$

where $\tilde{h}_{i}(e(t), e(t-\tau), r(t))=h_{i}(x(t), x(t-\tau), r(t))-h_{i}(s(t), s(t-\tau), r(t)), r(t)=\hat{i} \in S, e_{i}(t)=$ $x_{i}(t)-s(t), e_{i}(t-\tau)=x_{i}(t-\tau)-s(t-\tau)$.

Assumption 3 [16] Let $0<\beta<1$ and $\lambda>0$, there exists a continuous function $g:[0, \infty) \rightarrow$ $[0, \infty)$ with $g(0)>0$, for any $0 \leq u \leq t$, such that

$$
g(t)-g(u) \leq-\lambda \int_{u}^{t} g^{\beta}(s) d s
$$

Assume that the initial condition of the network (1) is given by

$$
x_{i}(z)=\varphi_{i}(z) \in \mathcal{C}\left([-\tau, 0], R^{n}\right), \quad i=1,2, \ldots, N
$$

where $\mathcal{C}\left([-\tau, 0], R^{n}\right)$ denotes the set of continuous functions mapping the interval $[-\tau, 0]$ into $R^{n}$.

Before ending this section, let us recall the following results, which will be used in the next section.

Lemma 1 (Finite-time stability theorem [25]) Suppose that function $V(t):[0, \infty) \rightarrow$ $[0, \infty)$ is differentiable (the derivative of $V(t)$ at 0 is in fact its right derivative) and $\frac{d V(t)}{d t} \leq-\eta V^{\beta}(t)$, where $\eta>0$ and $0<\beta<1$. Then $V(t)$ will reach zero at finite time $t^{*} \leq V^{1-\beta}(0) /(\eta(1-\beta))$ and $V(t)=0$ for all $t \geq t^{*}$.

Lemma 2 (Jesen inequality [26]) If $a_{1}, a_{2}, \ldots, a_{n}$ are positive number and $0<\alpha<\beta$, then $\left(\sum_{i=1}^{n} a_{i}^{\beta}\right)^{\frac{1}{\beta}} \leq\left(\sum_{i=1}^{n} a_{i}^{\alpha}\right)^{\frac{1}{\alpha}}$.

Lemma 3 [27] If $\xi_{1}, \xi_{2}, \ldots, \xi_{n} \geq 0$ and $0<p \leq 1$, then $\left(\sum_{i=1}^{n} \xi_{i}\right)^{p} \leq \sum_{i=1}^{n} \xi_{i}^{p}$.

\section{Main results}

In this section, we deal with the synchronization problem in finite time for the Markovian jump complex networks with time delays by designing pinning controller. By constructing new stochastic Lyapunov-Krasovskii functionals and using the finite-time stability theo- 
rem, sufficient conditions for the finite-time synchronization control problems are derived.

We present the finite-time synchronization criterion to ensure that a delayed complex network can be pinned. Subtracting (3) from (1), we obtain the following error dynamical systems:

$$
\begin{aligned}
\dot{e}_{i}(t)= & F\left(e_{i}(t), e_{i}(t-\tau)\right)+c \sum_{j=1}^{N} a_{i j}^{\hat{i}} \Gamma e_{j}(t)+u_{i}(t) \\
& +\tilde{h}_{i}(e(t), e(t-\tau), \hat{i}) d \omega_{i}(t), \quad i=1,2, \ldots, N, \hat{i} \in S,
\end{aligned}
$$

where $F\left(e_{i}(t), e_{i}(t-\tau)\right)=f\left(x_{i}(t), x_{i}(t-\tau)\right)-f(s(t), s(t-\tau)), \tilde{h}_{i}(e(t), e(t-\tau), \hat{i})=h_{i}(x(t), x(t-$ $\tau), \hat{i})-h_{i}(s(t), s(t-\tau), \hat{i}), e_{i}(t)=x_{i}(t)-s(t)$.

For the coupled system (9), we use the following linear negative feedback controllers:

$$
u_{i}(t)=-\varepsilon_{i}^{\hat{i}} \Gamma e_{i}(t)-k_{i}^{\hat{i}} \operatorname{sign}\left(e_{i}(t)\right)\left|e_{i}(t)\right|^{\beta},
$$

where $\varepsilon_{i}^{\hat{i}}>0$ and $k_{i}^{\hat{i}}>0, i \in J$, otherwise, $\varepsilon_{i}^{\hat{i}}=k_{i}^{\hat{i}}=0, i \notin J, \hat{i} \in S .\left|e_{i}(t)\right|^{\beta}=\left(\left|e_{i 1}(t)\right|^{\beta},\left|e_{i 2}(t)\right|^{\beta}\right.$, $\left.\ldots,\left|e_{i n}(t)\right|^{\beta}\right)^{T}$ and $\operatorname{sign}(\cdot)$ is the sign function, $\operatorname{sign}\left(e_{i}(t)\right)=\operatorname{diag}\left\{\operatorname{sign}\left(e_{i 1}(t)\right), \operatorname{sign}\left(e_{i 2}(t)\right)\right.$, $\left.\ldots, \operatorname{sign}\left(e_{i n}(t)\right)\right\}$, the real number $\beta$ satisfies $0<\beta<1, i=1,2, \ldots, N$.

In view of the pinning algorithm (10), we apply a pinning controller to the network (9) such that the network (1) has finite-time synchronization in the finite time. We obtain the following error dynamical systems:

$$
\begin{aligned}
\dot{e}_{i}(t)= & F\left(e_{i}(t), e_{i}(t-\tau)\right)+c \sum_{j=1}^{N} a_{i j}^{\hat{i}} \Gamma e_{j}(t)-\varepsilon_{i}^{\hat{i}} \Gamma e_{i}(t)-k_{i}^{\hat{i}} \operatorname{sign}\left(e_{i}(t)\right)\left|e_{i}(t)\right|^{\beta} \\
& +\tilde{h}_{i}(e(t), e(t-\tau), \hat{i}) d \omega_{i}(t), \quad i=1,2, \ldots, N
\end{aligned}
$$

where $\varepsilon_{i}^{\hat{i}}>0$ and $k_{i}^{\hat{i}}>0, i \in J$, otherwise, $\varepsilon_{i}^{\hat{i}}=k_{i}^{\hat{i}}=0, i \notin J, \hat{i} \in S . F\left(e_{i}(t), e_{i}(t-\tau)\right)=$ $f\left(x_{i}(t), x_{i}(t-\tau)\right)-f(s(t), s(t-\tau)), e_{i}(t)=x_{i}(t)-s(t)$.

Theorem 1 Let Assumptions 1-3 hold. If

$$
\left\{\begin{array}{l}
\frac{\left(1-\sigma+\bar{a}+\pi_{i}\right) I_{N}+\Psi^{\hat{i}}}{\lambda_{\min }(\Gamma)}+2 c A^{\hat{i}}-2 \Upsilon^{\hat{i}} \leq 0, \\
\Omega^{\hat{i}}-(1-\sigma-\bar{b}) I_{N} \leq 0, \\
q_{\hat{j}}-\delta_{\hat{i}} \leq 0, \quad \text { if } \hat{j} \neq \hat{i}, \hat{j} \in S, \\
q_{\hat{j}}-\delta_{\hat{i}} \geq 0, \quad \text { if } \hat{j}=\hat{i}, \hat{j} \in S,
\end{array}\right.
$$

where $\Upsilon^{\hat{i}}=\operatorname{diag}\{\underbrace{0, \ldots, 0}_{l}, \varepsilon_{l+1}^{\hat{i}}, \ldots, \varepsilon_{N}^{\hat{i}}\} \in R^{N \times N}$ is the diagonal matrix, $\pi_{\hat{i}}=\sum_{\hat{j} \in S_{1}^{\hat{i}}} \pi_{\hat{i}}\left(q_{\hat{j}}-\right.$ $\left.\delta_{\hat{i}}\right) / q_{\hat{i}}\left(\delta_{\hat{i}}>0, q_{\hat{i}}>1\right), 0<\sigma<1$, then, under the set of controllers (10), the complex network (1) is synchronization at finite time

$$
t^{*} \leq \tau+\frac{V(0, r(0))^{1-\frac{1+\beta}{2}}}{\gamma\left(1-\frac{1+\beta}{2}\right)},
$$


where $0<\beta<1, \gamma=\min (2 k, \lambda \sigma), k=\min _{i, \hat{i}}\left(k_{i}^{\hat{i}}\right), i \in J, \hat{i} \in S . V(0, r(0))=q_{r(0)} \sum_{i=1}^{N} e_{i}^{T}(0) e_{i}(0)$, $e_{i}(0)$ is the initial condition satisfying Assumption 2.

Proof For $\hat{i} \in S$, design the stochastic Lyapunov-Krasovskii functional $V(t, e(t), \hat{i})$ as follows:

$$
V(t, e(t), \hat{i})=q_{\hat{i}}\left[\sum_{i=1}^{N} e_{i}^{T}(t) e_{i}(t)+\sum_{i=1}^{N} \int_{t-\tau}^{t} e_{i}^{T}(s) e_{i}(s) d s\right],
$$

where $q_{\hat{i}}>1$.

Let $\mathcal{L} V$ (see [17], the generalized Itô formula) denote the second-order differential operator of $V$ with respect to (11) defined by

$$
\begin{aligned}
\mathcal{L} V(t, e(t), \hat{i})= & V_{t}(t, e(t), \hat{i})+V_{e}(t, e(t), \hat{i})\left[F\left(e_{i}(t), e_{i}(t-\tau)\right)\right. \\
& \left.+c \sum_{j=1}^{N} a_{i j}^{\hat{i}} \Gamma e_{j}(t)+u_{i}(t)\right]+\sum_{p \in S} \pi_{\hat{i}} V(t, e(t), \hat{j}) \\
& +\frac{1}{2} \operatorname{trace}\left[\tilde{h}_{i}^{T}(e(t), e(t-\tau), r(t)) V_{e e} \tilde{h}_{i}(e(t), e(t-\tau), r(t))\right] \\
= & 2 q_{\hat{i}} \sum_{i=1}^{N} e_{i}^{T}(t)\left[f\left(x_{i}(t), x_{i}(t-\tau)\right)-f\left(s_{l}(t), s_{l}(t-\tau)\right)\right. \\
& \left.+c \sum_{j=1}^{N} a_{i j}^{\hat{i}} \Gamma e_{j}(t)-\varepsilon_{i}^{\hat{i}} \Gamma e_{i}(t)-k_{i}^{\hat{i}} \operatorname{sign}\left(e_{i}(t)\right)\left|e_{i}(t)\right|^{\beta}\right] \\
& +q_{\hat{i}} \sum_{i=1}^{N} \operatorname{trace}\left[\tilde{h}_{i}^{T}(e(t), e(t-\tau), r(t)) \tilde{h}_{i}(e(t), e(t-\tau), r(t))\right] \\
& +q_{\hat{i}}\left(\sum_{i=1}^{N} e_{i}^{T}(t) e_{i}(t)-\sum_{i=1}^{N} e_{i}^{T}(t-\tau) e_{i}(t-\tau)\right) \\
& +\sum_{\hat{j} \in S} \pi_{\hat{i} \hat{j}} q_{\hat{j}}\left(\sum_{i=1}^{N} e_{i}^{T}(t) e_{i}(t)+\sum_{i=1}^{N} \int_{t-\tau}^{t} e_{i}^{T}(s) e_{i}(s) d s\right) .
\end{aligned}
$$

In view of Assumption 1, and the fact that $2 \mu|x y| \leq \mu^{2 \epsilon} x^{2}+\mu^{2(1-\epsilon)} y^{2}$, for $\forall \mu>0, x, y \in R$, $\epsilon \in[0,1]$, it is not difficult to get

$$
\begin{aligned}
& \left.2 \theta_{i j} e_{i}^{T}(t) e_{j}(t) \leq \theta_{i j}^{2 \epsilon} e_{i}^{2}(t)+\theta_{i j}^{2(1-\epsilon}\right) e_{j}^{2}(t), \\
& \left.2 \varphi_{i j} e_{i}^{T}(t) e_{j}(t-\tau) \leq \varphi_{i j}^{2 \epsilon} e_{i}^{2}(t)+\varphi_{i j}^{2(1-\epsilon}\right) e_{j}^{2}(t-\tau) .
\end{aligned}
$$

According to $\sum_{\hat{j} \in S} \pi_{\hat{i j}}=0$, for $\forall \delta_{\hat{i}}>0(\hat{i} \in S)$, it is easy to get $\left(\sum_{\hat{j} \in S_{1}^{\hat{i}}} \pi_{\hat{i}}+\sum_{\hat{j} \in S_{2}^{\hat{i}}} \pi_{\hat{i}}\right) \delta_{\hat{i}}=0$.

Denote $k=\min _{i, \hat{i}}\left(k_{i}^{\hat{i}}\right)(i \in J), \pi_{\hat{i}}=\sum_{\hat{j} \in \hat{S}_{1}^{\hat{i}}} \pi_{\hat{i}}\left(q_{\hat{j}}-\delta_{\hat{i}}\right) / q_{\hat{i}}, \xi_{i}^{\hat{i}}=\sum_{j=1}^{N} \xi_{i j}^{\hat{i}}, \eta_{i}^{\hat{i}}=\sum_{j=1}^{N} \eta_{i j}^{\hat{i}}, i=$ $1,2, \ldots, N$, and $\Psi^{\hat{i}}=\operatorname{diag}\left\{\xi_{1}^{\hat{i}}, \xi_{2}^{\hat{i}}, \ldots, \xi_{N}^{\hat{i}}\right\}, \Omega^{\hat{i}}=\operatorname{diag}\left\{\eta_{1}^{\hat{i}}, \eta_{2}^{\hat{i}}, \ldots, \eta_{N}^{\hat{i}}\right\}, \hat{i} \in S$. Let $0<\sigma<1$, from 
Assumption 2, the following inequality is obtained:

$$
\begin{aligned}
\mathcal{L} V(t, e(t), \hat{i}) \leq & q_{\hat{i}} e^{T}(t)\left[\left(\frac{\left(1-\sigma+\bar{a}+\pi_{\hat{i}}\right) I_{N}+\Psi^{\hat{i}}}{\lambda_{\min }(\Gamma)}+2 c A^{\hat{i}}-2 \Upsilon^{\hat{i}}\right) \otimes \Gamma\right] e(t) \\
& -2 k q_{\hat{i}} \sum_{i=1}^{N} \sum_{j=1}^{n}\left|e_{i j}(t)\right|^{1+\beta}+\sum_{\hat{j} \in S_{2}^{\hat{i}}} \sum_{i=1}^{N} \pi_{\hat{i}}\left(q_{\hat{j}}-\delta_{\hat{i}}\right) e_{i}^{T}(t) e_{i}(t) \\
& +q_{\hat{i}} e^{T}(t-\tau)\left[\left(\Omega^{\hat{i}}-(1-\sigma-\bar{b}) I_{N}\right) \otimes I_{n}\right] e(t-\tau) \\
& +\sum_{\hat{j} \in S} \pi_{\hat{i} \hat{j}}\left(q_{\hat{j}}-\delta_{\hat{i}}\right) \sum_{i=1}^{N} \int_{t-\tau}^{t} e_{i}^{T}(s) e_{i}(s) d s \\
& +q_{\hat{i}} \sigma\left(\sum_{i=1}^{N} e_{i}^{T}(t) e_{i}(t)-\sum_{i=1}^{N} e_{i}^{T}(t-\tau) e_{i}(t-\tau)\right) .
\end{aligned}
$$

In view of Assumption 3, let $\lambda>0,0<\frac{1+\beta}{2}<1, t \geq \tau$, and it follows that

$$
\begin{gathered}
\sigma\left(\sum_{i=1}^{N} q_{\hat{i}} e_{i}^{T}(t) e_{i}(t)-\sum_{i=1}^{N} q_{\hat{i}} e_{i}^{T}(t-\tau) e_{i}(t-\tau)\right) \\
\leq-\lambda \sigma \sum_{i=1}^{N} \int_{t-\tau}^{t}\left(q_{\hat{i}} e_{i}^{T}(s) e_{i}(s)\right)^{\frac{1+\beta}{2}} d s .
\end{gathered}
$$

By Lemma 2, we can see that, for $q_{\hat{i}}>1$,

$$
\left(q_{\hat{i}} \sum_{i=1}^{N} \sum_{j=1}^{n}\left|e_{i j}(t)\right|^{1+\beta}\right)^{\frac{1}{1+\beta}} \geq\left(q_{\hat{i}} \sum_{i=1}^{N} \sum_{j=1}^{n}\left|e_{i j}(t)\right|^{2}\right)^{\frac{1}{2}} .
$$

Thus,

$$
q_{\hat{i}} \sum_{i=1}^{N} \sum_{j=1}^{n}\left|e_{i j}(t)\right|^{1+\beta} \geq\left(q_{\hat{i}} \sum_{i=1}^{N} \sum_{j=1}^{n}\left|e_{i j}(t)\right|^{2}\right)^{\frac{1+\beta}{2}}=\left(q_{\hat{i}} \sum_{i=1}^{N} e_{i}^{T}(t) e_{i}(t)\right)^{\frac{1+\beta}{2}} .
$$

By Lemma 3, it is easy to get

$$
\sum_{i=1}^{N} \int_{t-\tau}^{t}\left(q_{\hat{i}} e_{i}^{T}(s) e_{i}(s)\right)^{\frac{1+\beta}{2}} d s \geq\left(q_{\hat{i}} \sum_{i=1}^{N} \int_{t-\tau}^{t} e_{i}^{T}(s) e_{i}(s) d s\right)^{\frac{1+\beta}{2}}
$$

It follows from the inequalities (15)-(17) that

$$
\begin{aligned}
\mathcal{L} V(t, e(t), \hat{i}) \leq & q_{\hat{i}} e^{T}(t)\left[\left(\frac{\left(1-\sigma+\bar{a}+\pi_{\hat{i}}\right) I_{N}+\Psi^{\hat{i}}}{\lambda_{\min }(\Gamma)}+2 c A^{\hat{i}}-2 \Upsilon^{\hat{i}}\right) \otimes \Gamma\right] e(t) \\
& -2 k\left(q_{\hat{i}} \sum_{i=1}^{N} e_{i}^{T}(t) e_{i}(t)\right)^{\frac{1+\beta}{2}}+\sum_{p \in S_{2}^{\hat{i}}} \sum_{i=1}^{N} \pi_{\hat{i}}\left(q_{\hat{j}}-\delta_{\hat{i}}\right) e_{i}^{T}(t) e_{i}(t)
\end{aligned}
$$




$$
\begin{aligned}
& +q_{\hat{i}} e^{T}(t-\tau)\left[\left(\Omega^{\hat{i}}-(1-\sigma-\bar{b}) I_{N}\right) \otimes I_{n}\right] e(t-\tau) \\
& +\sum_{\hat{j} \in S} \pi_{\hat{i} j}\left(q_{\hat{j}}-\delta_{\hat{i}}\right) \sum_{i=1}^{N} \int_{t-\tau}^{t} e_{i}^{T}(s) e_{i}(s) d s \\
& -\lambda \sigma\left(q_{\hat{i}} \sum_{i=1}^{N} \int_{t-\tau}^{t} e_{i}^{T}(s) e_{i}(s) d s\right)^{\frac{1+\beta}{2}} .
\end{aligned}
$$

Denote $\gamma=\min (2 k, \lambda \sigma)$, by Lemma 3, the following inequalities are given:

$$
\begin{aligned}
& 2 k\left(q_{\hat{i}} \sum_{i=1}^{N} e_{i}^{T}(t) e_{i}(t)\right)^{\frac{1+\beta}{2}}+\lambda \sigma\left(q_{\hat{i}} \sum_{i=1}^{N} \int_{t-\tau}^{t} e_{i}^{T}(s) e_{i}(s) d s\right)^{\frac{1+\beta}{2}} \\
& \geq \gamma\left[\left(q_{\hat{i}} \sum_{i=1}^{N} e_{i}^{T}(t) e_{i}(t)\right)^{\frac{1+\beta}{2}}+\left(q_{\hat{i}} \sum_{i=1}^{N} \int_{t-\tau}^{t} e_{i}^{T}(s) e_{i}(s) d s\right)^{\frac{1+\beta}{2}}\right] \\
& \geq \gamma\left[q_{\hat{i}}\left(\sum_{i=1}^{N} e_{i}^{T}(t) e_{i}(t)+\sum_{i=1}^{N} \int_{t-\tau}^{t} e_{i}^{T}(s) e_{i}(s) d s\right)\right]^{\frac{1+\beta}{2}} .
\end{aligned}
$$

Taking the expectation on both sides of (18), from (19) we get

$$
\begin{aligned}
\mathbf{E}[\mathcal{L} V(t, e(t), \hat{i})] \leq & \mathbf{E}\left\{q_{\hat{i}} e^{T}(t)\left[\left(\frac{\left(1-\sigma+\bar{a}+\pi_{\hat{i}}\right) I_{N}+\Psi^{\hat{i}}}{\lambda_{\min }(\Gamma)}+2 c A^{\hat{i}}-2 \Upsilon^{\hat{i}}\right) \otimes \Gamma\right] e(t)\right. \\
& +\sum_{\hat{j} \in S_{2}^{\hat{i}}} \sum_{i=1}^{N} \pi_{\hat{i}}\left(q_{\hat{j}}-\delta_{\hat{i}}\right) e_{i}^{T}(t) e_{i}(t) \\
& -\gamma\left[q_{\hat{i}}\left(\sum_{i=1}^{N} e_{i}^{T}(t) e_{i}(t)+\sum_{i=1}^{N} \int_{t-\tau}^{t} e_{i}^{T}(s) e_{i}(s) d s\right)\right]^{\frac{1+\beta}{2}} \\
& +q_{\hat{i}} e^{T}(t-\tau)\left[\left(\Omega^{\hat{i}}-(1-\sigma-\bar{b}) I_{N}\right) \otimes I_{n}\right] e(t-\tau) \\
& \left.+\sum_{\hat{j} \in S} \pi_{\hat{i}}\left(q_{\hat{j}}-\delta_{\hat{i}}\right) \sum_{i=1}^{N} \int_{t-\tau}^{t} e_{i}^{T}(s) e_{i}(s) d s\right\} .
\end{aligned}
$$

According to (12), one obtains $\mathbf{E}[\mathcal{L} V(t, e(t), \hat{i})] \leq-\gamma \mathbf{E}\left[V^{\frac{1+\beta}{2}}(t)\right]$. For any $t_{0}>0$, there is $\mathbf{E}\left[V^{\frac{1+\alpha}{2}}\left(t_{0}\right)\right]=\left(\mathbf{E}\left[V\left(t_{0}\right)\right]\right)^{\frac{1+\beta}{2}}$. Therefore, $\mathbf{E}[\mathcal{L} V(t, e(t), \hat{i})] \leq-\gamma(\mathbf{E}[V(t)])^{\frac{1+\beta}{2}}$. By Lemma 1, $\mathbf{E}[V(t)]$ converges to zero in a finite time, and the finite time is estimated by $t^{*} \leq \tau+$ $\frac{V(0)^{1-\frac{1+\beta}{2}}}{\gamma\left(1-\frac{1+\beta}{2}\right)}$.

Hence, the error vector $e_{i}(t)(i=1,2, \ldots, N)$ will stochastically converge to zero within $t^{*}$. According to the Definition 1, the coupled complex network (1) is finite-time synchronization in the finite time $t^{*}$. The proof is hence completed.

Remark 2 The model in this paper is more practical, because the network model involves time delays, stochastic perturbations, and partially known/unknown transition rates, while the models in $[11,12]$ did not contain time delays and partially known/unknown transition rates. The literature [14] did not consider the above three items; see Table 1. 
Table 1 Model comparisons

\begin{tabular}{lllll}
\hline Models & This paper & [11] & [12] & [14] \\
\hline Time delays & $\sqrt{ }$ & $\times$ & $\times$ & $\times$ \\
Stochastic perturbations & $\sqrt{ }$ & $\sqrt{ }$ & $\sqrt{ }$ & $\times$ \\
Partially unknown transition rates & $\sqrt{ }$ & $\times$ & $\times$ & $\times$ \\
\hline
\end{tabular}

Here ' $\sqrt{ }$ ' means that the model contains this component, ' $x$ ' means that the model does not contain this component.

Remark 3 [13-15, 24, 25] Investigated the finite-time boundedness synchronization problems for complex networks with time delays. Different from this literature, this paper studied the network synchronization to achieve in a finite time. Therefore, the result of this paper shows more advantages.

When $A(r(t))=A$, and we use the following linear negative feedback controllers:

$$
u_{i}(t)=-\varepsilon_{i} \Gamma e_{i}(t)-k_{i} \operatorname{sign}\left(e_{i}(t)\right)\left|e_{i}(t)\right|^{\beta},
$$

where $\varepsilon_{i}>0, k_{i}>0, i \in J$, otherwise, $\varepsilon_{i}=k_{i}=0, i \notin J$. $\left|e_{i}(t)\right|^{\beta}=\left(\left|e_{i 1}(t)\right|^{\beta},\left|e_{i 2}(t)\right|^{\beta}, \ldots\right.$, $\left.\left|e_{i n}(t)\right|^{\beta}\right)^{T}, i=1,2, \ldots, N$. We can obtain the following corollary.

Corollary 1 Let Assumptions 1 and 3 hold. If

$$
\left\{\begin{array}{l}
\frac{(1-\sigma+\bar{a}) I_{N}}{\lambda_{\min }(\Gamma)}+2 c A-2 \Upsilon \leq 0 \\
1-\sigma-\bar{b} \geq 0
\end{array}\right.
$$

where $\Upsilon=\operatorname{diag}\{\underbrace{0, \ldots, 0}_{l}, \varepsilon_{l+1}, \ldots, \varepsilon_{N}\} \in R^{N \times N}$ is the diagonal matrix, $1<l<N, 0<\sigma<1$, then, under the set of controllers (20), the complex network $(1)(A(r(t))=A)$ is synchronization at finite time $t^{*} \leq \tau+\frac{V(0)^{1-\frac{1+\beta}{2}}}{\gamma\left(1-\frac{1+\beta}{2}\right)}$, where $0<\beta<1, \gamma=\min (2 k, \lambda \sigma)$, and $k=\min _{i}\left(k_{i}\right)$, $i \in J . V(0)=\sum_{i=1}^{N} e_{i}^{T}(0) e_{i}(0), e_{i}(0)$ is the initial condition satisfying Assumption 2 of $e_{i}(t)=$ $x_{i}(t)-s(t), i=1,2, \ldots, N$.

\section{Numerical examples}

In this section, the example is given to demonstrate the effectiveness of the proposed approach.

Example 1 Consider the following Markovian jumping time-delay complex network:

$$
\begin{aligned}
\dot{x}_{i}(t)= & f\left(x_{i}(t), x_{i}(t-\tau)\right)+c \sum_{j=1}^{3} a_{i j}^{\hat{i}} \Gamma x_{j}(t)+u_{i}(t) \\
& +h_{i}(x(t), x(t-\tau), r(t)) d \omega_{i}(t),
\end{aligned}
$$

where $x_{i}(t)=\left(x_{i 1}(t), x_{i 2}(t)\right)^{T}$ is the state variable of the $i$ th node, $i=1,2,3, \hat{i}=1,2,3,4, \tau=1$, $c=1, \Gamma=\operatorname{diag}\{2,2.5\}$. We choose control nodes set $J=\{2,3\}$. We have

$$
A^{1}=\left[\begin{array}{ccc}
-8 & 7.98 & 0.02 \\
5.99 & -6 & 0.01 \\
0.01 & 5.49 & -5.5
\end{array}\right], \quad A^{2}=\left[\begin{array}{ccc}
-6.5 & 6.49 & 0.01 \\
4.18 & -4.2 & 0.02 \\
0.01 & 5.99 & -6
\end{array}\right]
$$




$$
\begin{aligned}
& A^{3}=\left[\begin{array}{ccc}
-4 & 3.99 & 0.01 \\
3.49 & -3.5 & 0.01 \\
0.01 & 4.99 & -5
\end{array}\right], \quad A^{4}=\left[\begin{array}{ccc}
-10 & 9.99 & 0.01 \\
8.49 & -8.5 & 0.01 \\
0.01 & 7.99 & -8
\end{array}\right], \\
& q_{1}=q_{2}=q_{3}=q_{4}=3 .
\end{aligned}
$$

The partially transition rate matrix is given by

$$
\left[\begin{array}{cccc}
-1 & ? & 0.2 & ? \\
0.6 & ? & ? & 0.2 \\
? & 0.3 & -2 & ? \\
? & ? & 0.1 & ?
\end{array}\right]
$$

The nonlinear function $f(\cdot)$ is given by [28]

$$
f\left(x_{i}(t), x_{i}(t-\tau)\right)=-C x_{i}(t)+A g\left(x_{i}(t)\right)+B g\left(x_{i}(t-\tau)\right)
$$

in which $g\left(x_{i}\right)=0.5\left(\left|x_{i 1}+1\right|-\left|x_{i 1}-1\right|,\left|x_{i 2}+1\right|-\left|x_{i 2}-1\right|\right)^{T}$, and matrices $C, A, B$ are, respectively, as follows:

$$
C=\left[\begin{array}{ll}
1 & 0 \\
0 & 1
\end{array}\right], \quad A=\left[\begin{array}{cc}
1+\frac{\pi}{4} & 20 \\
0.1 & 1+\frac{\pi}{4}
\end{array}\right], \quad B=\left[\begin{array}{cc}
-1.3 \sqrt{2} \frac{\pi}{4} & 0.1 \\
0.1 & -1.3 \sqrt{2} \frac{\pi}{4}
\end{array}\right] .
$$

Let $\epsilon=0.5$, we have $\bar{a}=11.6074$, and $\bar{b}=0.7720$ in view of (6) and the parameters of network (20). Now we verify that the condition (7) in Assumption 2 is satisfied. For $i=$ $1,2,3$, let $h_{i}^{\hat{i}}\left(t, x(t), x_{i}(t-\tau)\right)=0.1 \hat{i} \operatorname{diag}\left\{\bar{x}_{i 1}, \bar{x}_{i 2}\right\}$, where $\bar{x}_{i 1}=x_{i 1}(t)+x_{i 1}(t-\tau)-\left(x_{i+1,1}(t)+\right.$ $\left.x_{i+1,1}(t-\tau)\right), \bar{x}_{i 2}=x_{i 2}(t)+x_{i 2}(t-\tau)-\left(x_{i+1,2}(t)+x_{i+1,2}(t-\tau)\right)$. It is easy to see that

$$
\begin{aligned}
& \operatorname{trace}\left(\left(\tilde{h}_{i}^{\hat{i}}(e(t), e(t-\tau), t)\right)^{T} \tilde{h}_{i}^{1}(e(t), e(t-\tau), t)\right) \\
& \quad \leq 0.01 \hat{i}^{2}\left(\left\|e_{i}(t)\right\|_{2}^{2}+\left\|e_{i+1}(t)\right\|_{2}^{2}+\left\|e_{i}(t-\tau)\right\|_{2}^{2}+\left\|e_{i+1}(t-\tau)\right\|_{2}^{2}\right) .
\end{aligned}
$$

Let $e_{N+1}(t)=e_{1}(t), e_{N+1}(t-\tau)=e_{1}(t-\tau)$, that is, $e_{4}(t)=e_{1}(t), e_{4}(t-\tau)=e_{1}(t-\tau)$, then it follows that $\Psi^{\hat{i}}=\Omega^{\hat{i}}=\hat{i}^{2} \operatorname{diag}\{0.01,0.01,0.01\}, \hat{i}=1,2,3,4$. By using the Matlab Toolbox, applying Theorem 1 to this example and the following feasible solutions can be obtained: $\sigma=0.0127$ and

$$
\begin{array}{ll}
\Upsilon^{1}=\operatorname{diag}\{0,317.3576,298.0261\}, & \Upsilon^{2}=\operatorname{diag}\{0,305.2733,288.8442\}, \\
\Upsilon^{3}=\operatorname{diag}\{0,331.0433,290.6523\}, & \Upsilon^{4}=\operatorname{diag}\{0,317.3029,291.1940\} .
\end{array}
$$

The initial conditions of the numerical simulations are taken as: $x_{1}(0)=(-4.6,-2.4)^{T}$, $x_{2}(0)=(-5.2,3.9)^{T}, x_{3}(0)=(3.8,-1.4)^{T}$. Through a simple computation, we get $V(0)=$ 85.57 and $t^{*}=\tau+\frac{85.57^{1-\frac{1+\beta}{2}}}{\gamma\left(1-\frac{1+\beta}{2}\right)}$. Take $\gamma=20, \beta=0.6, \tau=1$, by computing we get $t^{*}=1.6087$. To further verify the effectiveness of the proposed control design for Example 1, Figure 1 describes the time response of the state errors variables $e_{12}(t), e_{13}(t), e_{23}(t)$, which implies that the addressed complex network (1) has achieved finite-time synchronization. 


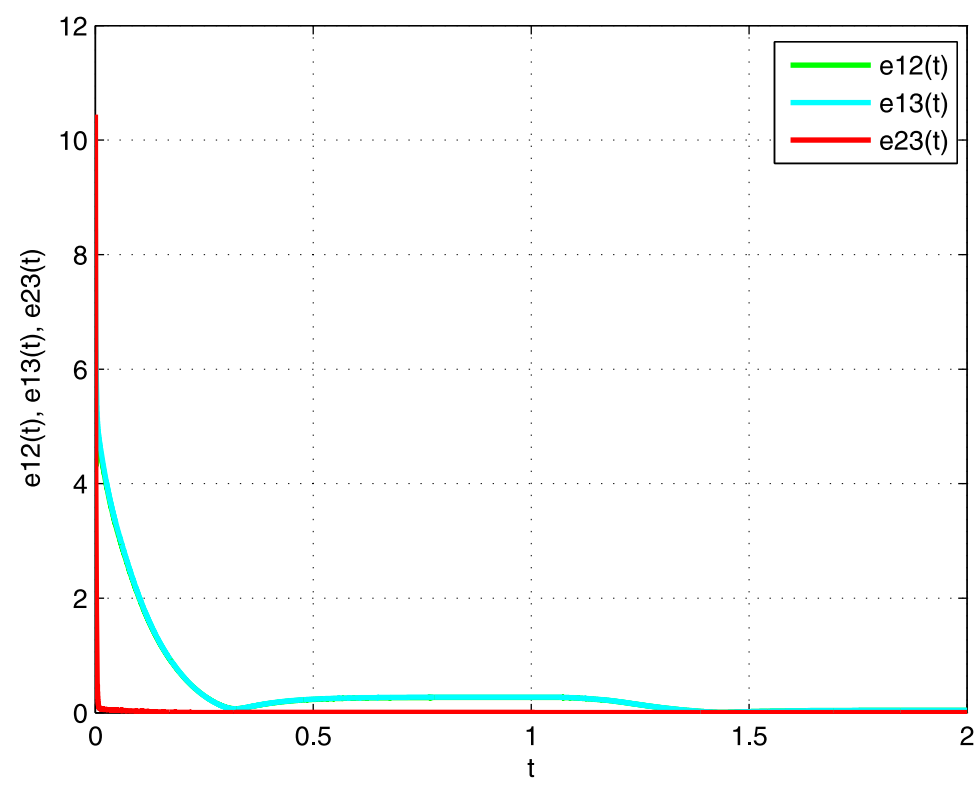

Figure 1 Time response of the error variables $e_{12}(t), e_{13}(t), e_{23}(t)$ for Example 1.

\section{Conclusions}

This paper has introduced a general delayed complex networks model with stochastic perturbations and the finite-time synchronization problem of Markovian switching complex networks with stochastic disturbance. Based on the finite-time stability theorem and inequality technique, easily testable conditions have been established to ensure finite-time synchronization for the addressed complex networks. Moreover, conditions that guarantee the finite-time synchronization of the delayed complex networks without switching have also been established. With variable time delays or random delays or mixed delays, finite-time synchronization research of Markovian switching complex network remains open. And it is hard for us to solve such problems, which is our future research.

\section{Competing interests}

The authors declare that they have no competing interests.

\section{Authors' contributions}

All authors contributed equally to the writing of this paper. All authors read and approved the final manuscript.

\section{Author details}

${ }^{1}$ College of Fundamental Studies, Shanghai University of Engineering Science, Shanghai, 201620, China. ${ }^{2}$ College of Information Science and Technology, Donghua University, Shanghai, 201620, China. ${ }^{3}$ Modern Industry Training Center, Shanghai University of Engineering Science, Shanghai, 201620, China.

\section{Acknowledgements}

The work was supported by the Education Commission Scientific Research Innovation Key Project of Shanghai under Grant 13ZZ050, the Science and Technology Commission Innovation Plan Basic Research Key Project of Shanghai under Grant 12JC1400400.

Received: 10 November 2013 Accepted: 20 March 2014 Published: 19 May 2014

\section{References}

1. Watts, DJ, Strogatz, SH: Collective dynamics of small-world. Nature 393, 440-442 (1998)

2. Li, H: Synchronization stability for discrete-time stochastic complex networks with probabilistic interval time-varying delays. Int. J. Innov. Comput. Inf. Control 7, 697-708 (2011)

3. $\mathrm{Wu}, \mathrm{Z}, \mathrm{Shi}, \mathrm{P}, \mathrm{Su}, \mathrm{H}, \mathrm{Chu}, \mathrm{J}$ : Sampled-data exponential synchronization of complex dynamical networks with time-varying coupling delay. IEEE Trans. Neural Netw. Learn. Syst. 24, 1177-1187 (2013) 
4. $\mathrm{Wu}, \mathrm{Z}, \mathrm{Shi}, \mathrm{P}, \mathrm{Su}, \mathrm{H}, \mathrm{Chu}, \mathrm{J}$ : Sampled-data synchronization of chaotic Lur'e systems with time delays. IEEE Trans. Neural Netw. Learn. Syst. 24, 410-421 (2013)

5. Wu, CW, Chua, LO: A unified framework for synchronization and control of dynamical systems. Int. J. Bifurc. Chaos 4, 979-998 (1994)

6. Lü, J, Yu, X, Chen, G: Synchronization of general complex dynamical networks. Physica A 334, 281-302 (2004)

7. Tang, Y, Wong, WK: Distributed synchronization of coupled neural networks via randomly occurring control. IEEE Trans. Neural Netw. Learn. Syst. 24, 435-447 (2013)

8. $\mathrm{Wu}, \mathrm{Z}, \mathrm{Shi}, \mathrm{P}, \mathrm{Su}, \mathrm{H}, \mathrm{Chu}, \mathrm{J}$ : Stochastic synchronization of Markovian jump neural networks with time-varying delay using sampled data. IEEE Trans. Cybern. 43, 1796-1806 (2013)

9. Zhang, W, Tang, Y, Fang, J, Zhu, W: Exponential cluster synchronization of impulsive delayed genetic oscillators with external disturbances. Chaos 21, 043137 (2011)

10. Yang, X, Cao, J, Lu, J: Synchronization of Markovian coupled neural networks with nonidentical node delays and random coupling strengths. IEEE Trans. Neural Netw. Learn. Syst. 1, 60-71 (2012)

11. Yang, X, Cao, J: Finite-time stochastic synchronization of complex networks. Appl. Math. Model. 34, 3631-3641 (2010)

12. Sun, Y, Li, W, Zhao, D: Finite-time stochastic outer synchronization between two complex dynamical networks with different topologies. Chaos 22, 023152 (2012)

13. $\mathrm{Ma}, \mathrm{Q}$, Wang, Z, Lu, J: Finite-time synchronization for complex dynamical networks with time-varying delays. Chaos 22, 043151 (2012)

14. Chen, Y, Lü, J: Finite-time synchronization of complex dynamical networks. J. Syst. Sci. Complex. 29, 1419-1430 (2009)

15. Hou, L, Zong, G, Wu, Y: Finite-time control for switched delay systems via dynamic output feedback. Int. J. Innov. Comput. Inf. Control 8, 4901-4913 (2012)

16. Cui, W, Fang, J, Zhang, W, Wang, X: Finite-time cluster synchronization of Markov switching complex networks with stochastic perturbations. IET Control Theory Appl. 8, 30-41 (2014)

17. Yuan, C, Mao, X: Robust stability and controllability of stochastic differential delay equations with Markovian switching. Automatica 40, 343-354 (2004)

18. $\mathrm{Wu}, \mathrm{Z}, \mathrm{Shi}, \mathrm{P}, \mathrm{Su}, \mathrm{H}, \mathrm{Chu}, \mathrm{J}$ : Passivity analysis for discrete-time stochastic Markovian jump neural networks with mixed time delays. IEEE Trans. Neural Netw. 22, 1566-1575 (2011)

19. Zhang, L, Boukas, E, Lam, J: Analysis and synthesis of Markov jump linear systems with time-varying delays and partially known transition probabilities. IEEE Trans. Autom. Control 53, 2458-2464 (2009)

20. Du, B, Lam, J, Zou, Y, Shu, Z: Stability and stabilization for Markovian jump time-delay systems with partially unknown transition rates. IEEE Trans. Circuits Syst. I, Regul. Pap. 60, 341-351 (2013)

21. Zhang, Y, He, Y, Wu, M, Zhang, J: Stabilization for Markovian jump systems with partial information on transition probability based on free-connection weighting matrices. Automatica 47, 79-84 (2011)

22. Xiong, J, Lam, J, Gao, H, Ho, D: On robust stabilization of Markovian jump systems with uncertain switching probabilities. Automatica 41, 897-903 (2005)

23. Shu, Z, Lam, J, Xu, S: Robust stabilization of Markovian delay systems with delay-dependent exponential estimates. Automatica 42, 2001-2008 (2006)

24. Yin, J, Khoo, S, Man, Z, Yu, X: Finite-time stability and instability of stochastic nonlinear systems. Automatica 47, 2671-2677 (2011)

25. Bhat, SP, Bernstein, DS: Finite-time stability of continuous autonomous systems. SIAM J. Control Optim. 38, 751-766 (2000)

26. Xu, L, Wang, X: Mathematical Analysis Methods and Examples, pp. 36-37. Higher Education Press, Beijing, China (1983)

27. Wang, L, Xiao, F: Finite-time consensus problems for networks of dynamic agents. IEEE Trans. Autom. Control 55, 950-955 (2010)

28. Gilli, M: Strange attractors in delayed cellular neural networks. IEEE Trans. Circuits Syst. I, Fundam. Theory Appl. 40 849-853 (1993)

10.1186/1687-1847-2014-149

Cite this article as: Cui et al.: Finite time synchronization problems of delayed complex networks with stochastic perturbations. Advances in Difference Equations 2014, 2014:149

\section{Submit your manuscript to a SpringerOpen ${ }^{\ominus}$ journal and benefit from:}

- Convenient online submission

Rigorous peer review

- Immediate publication on acceptance

- Open access: articles freely available online

- High visibility within the field

- Retaining the copyright to your article

Submit your next manuscript at $\gg$ springeropen.com 